EPJ Web of Conferences 41, 01008 (2013)

DOI: $10.1051 /$ epjconf/20134101008

(C) Owned by the authors, published by EDP Sciences, 2013

\title{
Polarization-controlled quasi-phase-matching of high harmonic generation
}

\author{
L. Z. Liu ${ }^{1}$, K O’Keeffe $^{1}$, S. M. Hooker ${ }^{1}$ \\ ${ }^{1}$ University of Oxford Physics Department, Parks Road, Oxford, UK OX1 3PU
}

\begin{abstract}
A new class of quasi-phase matching high harmonic generation is proposed where the polarization of the driving field is controlled in a waveguide. The first circularly polarized quasi-phase matched source is shown to be possible.
\end{abstract}

\section{INTRODUCTION}

High harmonic generation (HHG) is a nonlinear process in which odd multiples of a fundamental driving field are produced when an intense laser pulse is focused into a low density gas. HHG is an attractive source of temporally and spatially coherent, tuneable light with wavelengths in the XUV and soft X-ray range and has found a broad range of applications in ultrafast physics and imaging. However, due to the phase mismatch between the driving and generated fields, the intensity of the generated harmonics oscillates as a function of propagation distance, $z$, between 0 and some maximum value. The period of oscillation is $2 \mathrm{~L}_{c}$ where $\mathrm{L}_{c}$ is the coherence length. One way of overcoming the problem of phasemismatching is quasi-phase matching (QPM), in which harmonic generation is suppressed in out-of phase regions. Here we propose a new class of QPM techniques by controlling the polarization in an optical waveguide by either: (i) rotating the polarization of the driving field [1,2] in a circularly birefringent waveguide; or (ii) modulating the ellipticity of the driver polarization $[3,4]$ in a linearly birefringent system. The key advantage of this new class of polarization-controlled QPM is its simplicity compared to other QPM techniques since it avoids the need for additional laser pulses [5] or longitudinally-structured waveguides [6]. Instead, the only requirement is a waveguide with a suitable value of birefringence. Moreover, as we show below, the circular birefringent scheme affords significantly greater conversion efficiencies than other QPM schemes and also provides the first circularly polarized quasi-phase matched harmonic source.

\section{Optical Rotation Quasi-Phase Matching (ORQPM)}

ORQPM utilizes a waveguide with circular birefringence, which causes the plane of polarization of linearly polarized light to rotate with propagation distance at a constant rate, with period $2 L_{r}$. By matching $L_{r}=L_{c}$, the generated harmonics will grow monotonically. Because the polarization of the driving field remains linear, harmonics are generated locally with the same amplitude at each point. Further, if $L_{r}=L_{c}$ at points were - in the absence of QPM - destructive interference would occur, such as at $z=L_{c}$, the phase of the driving field is flipped by $\pi$, and hence the locally-generated harmonics remain in phase with those generated earlier. Based on this, the harmonic intensity of the $q$ th harmonic can be written as:

This is an Open Access article distributed under the terms of the Creative Commons Attribution License 2.0, which permits unrestricted use, distribution, and reproduction in any medium, provided the original work is properly cited. 


$$
\mathrm{I}_{\mathrm{q}}(\mathrm{z})=\mathrm{A}^{2}\left\{1 / 2 \mathrm{z}^{2}+1-[\cos (2 \Delta \mathrm{kz})] /\left[4(\Delta \mathrm{k})^{2}\right]\right\}
$$

where $\mathrm{A}$ is a normalization factor. We see that the growth of the harmonic intensity comprises a quadratic term, which dominates at large $\mathrm{z}$, plus a weak cosinusoidal modulation. It is clear that in the limit of large $\mathrm{z}$, ORQPM is half as efficient as would be true phase-matching-i.e., setting $\Delta \mathrm{k}=0$ - under otherwise identical conditions. Moreover, ORQPM is approximately 5 times more efficient than ideal square-wave QPM modulation. Finally, ORQPM is the first QPM or phase matching scheme to generate circularly polarized high harmonics, where the harmonics will have the same handedness as the rotation of the driving field.

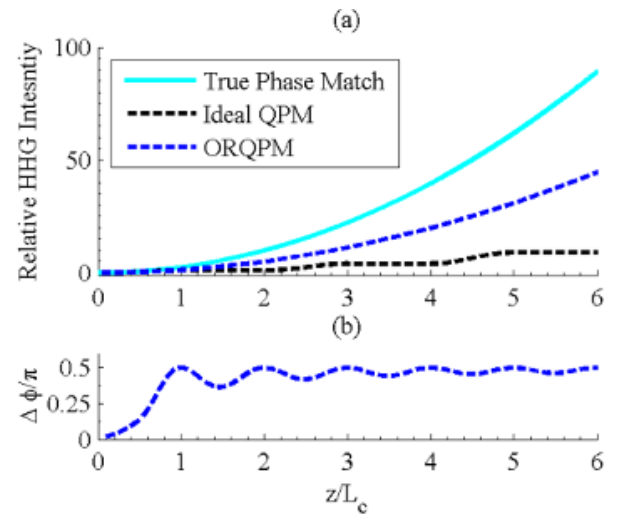

Fig. 1.Plot (a) shows the harmonic intensity as a function of $z$ for true phase matching, ideal QPM, and ORQPM. Plot (b) shows the phase difference between the $\mathrm{x}$ - and $\mathrm{y}$ - polarization components.

Simulations, assuming a continuous wave envelope approximation, were undertaken. Fig. 1a shows the calculated harmonic intensity for ORQPM compared with true-phase matching and perfect QPM with a square-wave modulation. We see that the ORQPM generates harmonics with an intensity much greater than for standard QPM, and comparable to that obtained with true-phase matching. Fig. 1b shows the phase difference between the $\mathrm{x}$ - and $\mathrm{y}$ - polarization components as a function of propagation distance. It can be seen that after approximately $5 L_{\mathrm{c}}$ the phase difference is approximately constant and equal to $\pi / 2$, corresponding to circular polarization.

\section{Polarization Beating Quasi-Phase Matching (PBQPM)}

It is well known that the single-atom efficiency of HHG depends sensitively on the polarization of the driving laser field [7] which arises from the fact that the ionized electron must return to the parent ion in order to emit a harmonic photon. In PBQPM, a birefringent waveguide is used to generate beating of the polarization state of a driving linearly-polarized driving laser pulse, thereby modulating the harmonic generation process. In a birefringent waveguide, the incident radiation can be resolved into two components polarized along the birefringent axes. For linearly-polarized incident light these components are initially in phase, but the difference in their phase velocity will cause the two components to develop a phase difference which increases linearly with propagation distance. As a consequence, the resultant polarization state will evolve from linearly polarized at an angle $\Theta$ to (for example) the fast axis, through (say) right-handed elliptical polarization to linearly polarized at an angle $-\Theta$ to the fast axis, and thence through elliptical polarization back to linearly-polarized radiation parallel to the incident light. QPM will occur if the period of polarization beating is suitably matched to the coherence length of the harmonics, where the generated harmonics will be linearly polarized. 


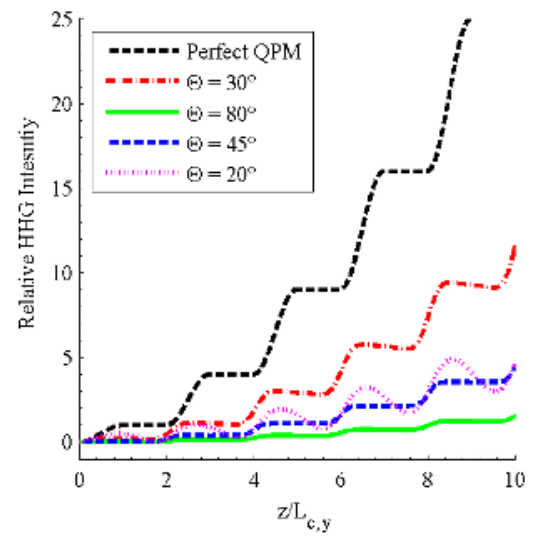

Fig. 2 Harmonic intensity as a function $z$ for different coupling angles $\Theta$, for $L_{b}=2 L_{c, y}$ for PBQPM.

Fig. 2 shows the harmonic intensity calculated for PBQPM for different coupling angles $\Theta$, defined by the angle the input polarization makes with the faster birefringent axis. Note that there is an optimum coupling angle: if $\Theta$ is too small, then the driver ellipticity remains small for all $\mathrm{z}$, and the harmonic generatio is not sufficiently suppressed in the out-of-phase zones; if it is too large then harmonics are generated for only a small fraction of the in-phase zones.

\section{Conclusion}

To achieve either polarization controlled QPM, one can implement a birefringent waveguide to modulate the polarization of the driving radiation - circular birefringence for ORQPM and linear birefringence for PBQPM. Such waveguide could be constructed with birefringent material or special geometries such as those employed in photonic crystal fibers. Alternatively the excitation of two orthogonally-polarized modes of a non-birefringent waveguide causes the driving radiation to beat between linear and elliptical, which can be employed for PBQPM. Similarly, coupling in two contra-rotating circularly polarized modes causes the driving radiation to rotate, which can be used for ORQPM. In conclusion we have proposed a novel method for QPM HHG, and have undertaken initial calculations aimed at understanding the optimal experimental conditions for ORQPM or PBQPM. We would like to thank the EPSRC Grant No. EP/GO67694/1 and the Buckee Scholarship of Merton College, Oxford.

\section{References}

1. L. Z. Liu, K. O'Keeffe, and S. M. Hooker, "High harmonic optical generator [Polarization Beating]," Isis Innovation, U.K. Patent Application No. B1117355.6 (07 Oct. 2011).

2. L. Z. Liu, K. O'Keeffe, and S. M. Hooker, "Quasi-phase-matching of high harmonic generation using polarization beating in optical waveguides," Phys. Rev. A, 85(5): 053823 (2012)

3. L. Z. Liu, K. O'Keeffe, and S. M. Hooker, "High harmonic optical generator [Optical Rotation]," Isis Innovation, U.K. Patent Application No. GB1208753.2 (18 May 2012).

4. L. Z. Liu, K. O'Keeffe, and S. M. Hooker, "Optical Rotation Quasi-Phase-Matching for Circularly Polarized High Harmonic Generation," Opt Lett, 37(12): 2415-2417 (2012)

5. X. Zhang, et al. "Quasi-phase-matching and quantum-path control of high-harmonic generation using counterpropagating light.” Nature Phys 3(4):270-275 (2007)

6. I. Christov et al "Quasi-phase matching of high harmonics and attosecond pulses in modulated waveguides." Opt. Express, 7(11):362-367 (2000)

7. K. S. Budil,et al. "Influence of ellipticity on harmonic generation,” Phys. Rev. A, 48(5): R3437-R3440 (1993) 